\title{
On thermodynamic states of the Ising model on scale-free graphs*
}

\author{
Yu. Kozitsky \\ Institute of Mathematics, Maria Curie-Sklodowska University, 20-031 Lublin, Poland
}

Received October 11, 2012, in final form November 2, 2012

There is proposed a model of scale-free random graphs which are locally close to the uncorrelated complex random networks with divergent $\left\langle k^{2}\right\rangle$ studied in, e.g., Dorogovtsev S.N. et al., Rev. Mod. Phys., 2008, 80, 1275. It is shown that the Ising model on the proposed graphs with interaction intensities of arbitrary signs with probability one is in a paramagnetic state at sufficiently high finite values of the temperature. For the same graphs, the bond percolation model with probability one is in a nonpercolative state for positive values of the percolation probability. These results and their possible extensions are also discussed.

Key words: random graphs, paramagnetic phase, percolation, branching process

PACS: 05.70.Fh, 05.50.+q, 02.50.Ga

\section{Introduction}

In statistical physics, a paradigm model for cooperative phenomena is the Ising model of interacting spins attached to the vertices of a graph $\mathrm{G}=(\mathrm{V}, \mathrm{E})$. This model is described by the Hamiltonian

$$
H=-\sum_{\{x, y\} \in \mathrm{E}} J_{x y} \sigma_{x} \sigma_{y}-h \sum_{x \in \mathrm{V}} \sigma_{x}
$$

where $J_{x y}$ are interaction intensities, $h$ is an external field, and the spins $\sigma_{x}$ take values \pm 1 . The sums run over the sets of edges $E$ and vertices $V$ of the graph $G$, respectively. The graph is supposed to be connected and countably infinite. In view of the latter fact, the Hamiltonian (1.1) has no direct mathematical meaning and is used as a formula for local Hamiltonians, $H_{\Lambda}$, which one obtains by restricting the sums to finite subsets $\Lambda \subset \mathrm{V}$ and $\mathrm{E}_{\Lambda} \subset \mathrm{E}\left(\mathrm{E}_{\Lambda}\right.$ being the collection of edges with both ends in $\Lambda$ ). Then, the thermodynamic properties of (1.1) are studied in the limit $\Lambda \rightarrow V$. In a more sophisticated theory [1], a thermodynamic state is a Gibbs measure, and a phase transition is associated with the possibility for multiple thermodynamic states to exist at the same values of the temperature and $h$.

Obviously, the properties of the model in (1.1) are closely related to those of the underlying graph. The case of a special interest is where this graph is random, with vertices having an arbitrary number of neighbors. Then, the graph itself can be characterized by the properties of the corresponding Ising model. Perhaps, In view of this fact methods of statistical physics are widely used also in the study of the underlying random graphs. A long list of publications regarding this topic can be found in the review articles [2, 3].

For a vertex $x$, the degree $k(x)$ is defined as the number of neighbors of $x$, i.e., the number of $y \in \mathrm{V}$ such that $\{x, y\} \in \mathrm{E}$. If $\mathrm{G}$ is random, the degree of each $x \in \mathrm{V}$ is an integer valued random variable and hence is characterized by the probability distribution

$$
\operatorname{Prob}\{k(x)=k\}=p_{k}(x), \quad k \geqslant 1, \quad x \in \mathrm{V} .
$$

\footnotetext{
${ }^{*}$ The paper is dedicated to Mykhailo Kozlovskii on the occasion of his 60-th birthday.
} 
In simple cases, the degrees $k(x)$ are independent and identically distributed, which particularly means that the probabilities $p_{k}(x)$ are the same for all $x$. If $p_{k}=C k^{-\lambda}$, at least for big enough $k$, the corresponding graph is said to be scale-free, cf. [4]. In this case, the $m$-th moment

$$
\left\langle k^{m}\right\rangle=\sum_{k \geqslant 1} k^{m} p_{k}, \quad m \geqslant 1
$$

exists provided $\lambda>m+1$. Notably, $\left\langle k^{2}\right\rangle=+\infty$ for many real-world networks [3].

An example of a random graph with independent $k(x)$ is a Galton-Watson tree, which is a genealogic tree of a Galton-Watson branching process [5, 6]. In more complex models, the graph $G$ is obtained, more or less explicitly, as the limit of an increasing sequence of finite random graphs, $\left(\mathrm{V}_{n}, \mathrm{E}_{n}\right), n \in \mathbb{N}$, see, e.g., [7-10]. In the physical literature, such constructions are rather informal which leaves certain possibilities for different interpretations. In a sense, the aim of this paper is to show that the lack of precision in defining the underlying random graph may yield different conclusions on the thermodynamic states of the corresponding Ising model. In 2002, almost simultaneously there appeared two papers [11, 12] where the model (1.1) on a scale-free graph was studied. The starting point is a scale-free distribution $\left\{p_{k}\right\}_{k \in \mathbb{N}}$, which "characterizes the entire net", whereas the "local structure of connections" is characterized by "the distribution of the number of connections of the nearest neighbor of a vertex", which is taken to be $k p_{k} /\langle k\rangle$, see the beginning of the first section in [11]. In [12], a similar assumption was made, see equation (3) therein, as well as (1) in [2]. In the latter article, such graphs are referred to as complex networks in contrast to the classical models - simple networks, one of which is presumably the Galton-Watson tree mentioned above. In both papers [11, 12], it was found that the model (1.1) with $h=0$, ferromagnetic interactions $J_{x y}=J>0$, and $\left\langle k^{2}\right\rangle=+\infty$ is in an ordered (ferromagnetic) state at all temperature $\frac{1}{1} T>0$ . Recently, in [9, 10] a mathematical construction of a random graph model was presented, in which the mentioned properties were realized. Then, it has become clear that the conclusions of [11, 12] repeat, in a sense, the results of [14], see subsection [5.2 below. In this work, we construct "uncorrelated complex networks” with $\left\langle k^{2}\right\rangle=\infty$, consistent with the assumptions made in [2, 11, 12] and mentioned above, for which the model 1.1 with interaction intensities of arbitrary signs can be in a paramagnetic state at finite temperatures. Note that the graph model which we propose here is only locally mathematically similar to that of [11, 12]. This means that our result does not contradict the conclusions of the latter papers because it applies to a different model. A more detailed discussion regarding this subject can be found in section 5 below.

In section 2 we construct the graph model which is a combination of the one used in [9, 10] and of the ordinary Galton-Watson tree. In section 3 we prove theorem 3.1 the main result of which is that the model (1.1) on the introduced graphs, with any signs of $J_{x y}$, can be in a paramagnetic state at finite temperatures and $h=0$, provided $\left\langle k^{1+\alpha}\right\rangle<\infty$ for some $\alpha>0$. This covers the case of scale-free distributions with $\lambda \in$ $(2,3)$. The proof of theorem 3.1 relies upon two lemmas, proven in section 4 The result of theorem 3.1 and the graph model introduced in section 2 are discussed in the concluding section 5 where we also present an extension of the results of [11, 12] concerning the model [1.1] with interaction intensities of arbitrary signs. Except possibly for section 4 the paper is self-contained and accessible to non-mathematicians.

\section{The graph models}

Since the Galton-Watson tree will serve us as a prototype, we begin by presenting this model. More on this topic can be found in [5, 6, 15].

\subsection{The Galton-Watson tree}

To exclude the possibility of obtaining a finite tree or a tree of a bounded degree, we suppose that the underlying random variable $X$ takes positive integer values $k \in \mathbb{N}$ with probability

$$
\operatorname{Prob}(X=k)=p_{k+1} \in[0,1)
$$

\footnotetext{
${ }^{1}$ In contrast to regular graphs, e.g., crystal lattices, the Ising model on sufficiently 'dense’ non-random graphs can be in an ordered state at all temperatures, see, e.g., the example in [13].
} 
such that

$$
\sum_{k \geqslant 2} p_{k}=1, \quad a:=\sum_{k \geqslant 2} k p_{k}<\infty, \quad \sum_{k=2}^{n} p_{k}<1, \quad \text { for any } \quad n \in \mathbb{N} \text {. }
$$

One observes that

$$
a>2 \text {, }
$$

which readily follows from the properties assumed in (2.1). Set $L_{0}=1, L_{1}=X$, and

$$
L_{n}=\sum_{j=1}^{L_{n-1}} X_{n, j},
$$

where all $X_{n, j}$ are independent copies of $X$, see chapters II and III in [5] or chapter 5 in [6]. Then, $L_{n}$ is the number of individuals in generation $n$ of a Galton-Watson branching process. The process starts with a single individual, and each individual in generation $n$ produces offsprings at random, independently and with the same distribution as $X$. The individuals existing in all generations form the vertex set $\mathrm{V}$ of the corresponding (genealogic) Galton-Watson graph $\mathrm{G}$, with undirected edges $\{x, y\} \in \mathrm{E}$ connecting each individual and its offsprings. Under the assumptions made in (2.1), the corresponding process is not extinct and $L_{n} \rightarrow+\infty$ with probability one. Hence, $G$ is an infinite tree - an acyclic connected graph, which has no leaves, except possibly for the root $o$. By construction, the vertex degrees are independent identically distributed random variables such that

$$
\operatorname{Prob}\{k(o)=k\}=p_{k+1}, \quad \operatorname{Prob}\{k(x)=k\}=p_{k}, \quad x \in \bigvee \backslash\{o\} .
$$

Now let us fix the graph-theoretical and probabilistic terminology used in this article. For a graph $\mathrm{G}=$ $(\mathrm{V}, \mathrm{E})$ and vertices $x, y \in \mathrm{V}$, a path $\vartheta(x, y)$ is a sequence $\left\{x_{0}, x_{1}, \ldots, x_{n}\right\}, n \in \mathbb{N}$, such that: (a) $x_{0}=x, x_{n}=y$; (b) $\left\{x_{l}, x_{l+1}\right\} \in \mathrm{E}$; (c) neither of $x_{0}, \ldots, x_{n-1}$ can be repeated. The length of the path is $\|\vartheta(x, y)\|=n$; $x$ is its origin and $y$ is terminus. A ray with the origin $x_{0}$ is an infinite sequence of distinct vertices $\left\{x_{0}, x_{1}, \ldots, x_{n}, \ldots\right\}$ with the property (b) as above. A graph ensemble $\mathscr{G}$ is the collection of all possible realizations as just described. A random graph model is a triple $(\mathscr{G}, \mathscr{F}, P)$, where $\mathscr{F}$ is a certain family of subsets of $\mathscr{G}$, and $P: \mathscr{F} \rightarrow[0,1]$ is the probability. For the Galton-Watson tree, $P$ is defined by the collection $p=\left\{p_{k}\right\}$. For instance, if $\mathscr{A}$ is the set of all realizations with $k(o)=1$, then $P(\mathscr{A})=p_{1}$. In general, for $\mathscr{A} \in \mathscr{F}, P(\mathscr{A})$ is the probability of the event: "the graph chosen at random from $\mathscr{G}$ belongs to $\mathscr{A}$ ". Events of a certain sort can occur with probability either zero or one - the celebrated zero-one law. We say that a property of a random graph holds with probability one if this property is possessed by all of the elements of some $\mathscr{A}$ such that $P(\mathscr{A})=1$. Usually, when one deals with the models with independent vertex degrees, the triple $(\mathscr{G}, \mathscr{F}, P)$ does not appear explicitly. This will also be the case in the rest of the paper.

\subsection{The configuration model}

Let $p:=\left\{p_{k}\right\}_{k \geqslant 2}$ be as in (2.1), and

$$
\hat{p}_{k}:=k p_{k} / a, \quad k \geqslant 2 .
$$

“Uncorrelated complex networks” were 'defined' in [2, 11, 12] by means of the following conditions. The main one is that "the neighbor of a vertex has a degree $k$ with probability $\hat{p}_{k}$ ", which can be interpreted as the condition that in every path $\left\{x_{0}, x_{1}, \ldots, x_{n}\right\}$, the probability that $k\left(x_{l}\right)$ takes value $k$ is $\hat{p}_{k}$. We shall call it a path property. Other important assumptions are: (a) all $k(x)$ are "uncorrelated" (in fact, independent); (b) two paths $\left\{x, x_{1}, \ldots, y\right\}$ and $\left\{x, x_{1}^{\prime}, \ldots, z\right\}$, with $x_{1} \neq x_{1}^{\prime}$ never intersect each other, i.e., the graph is a tree 2 . These assumptions are consistent with the following mathematical models. The first one is the ordinary Galton-Watson tree with $p$ replaced by $\hat{p}$, in [9] it is $\mathrm{T}(\rho, \infty)$, see page 567 thereof. A slightly

\footnotetext{
${ }^{2}$ In [2, 11], the graphs were called "tree-like" if the intersections are negligible. Mathematical definitions of tree-like graphs can be found, e.g., in $[9,10,14]$.
} 
different version is the so-called configuration model, cf. page 1279 in [2]. This model is a rooted tree with independent vertex degrees such that

$$
\operatorname{Prob}(k(o)=k)=p_{k}, \quad \operatorname{Prob}(k(x)=k)=\hat{p}_{k} .
$$

This mode $\sqrt[3]{3}$ was studied in [9, 10], in [9] it appears as $\mathrm{T}(P, \rho, \infty)$. The main characteristic feature of both $\mathrm{T}(P, \rho, \infty)$ and $\mathrm{T}(\rho, \infty)$ is that each path $\vartheta(x, y), x \neq o$, has the path property described above. The inconsistency with the root degrees does not change the global behavior of the graph, and hence of the corresponding model (1.1).

The construction of the configuration model can be visualized as follows. Consider a countable set of 'points' (a configuration), each of which is given a random marl] $k \in \mathbb{N}$ with probability distribution $p$ as in (2.1). The marks of different points are independent. Then, the graph is obtained as follows. The root degree is drawn from $\mathbb{N}$ according to $\operatorname{Prob}(k(o)=k)=p_{k}$. If the result is $m$, the root receives $m$ neighbors - 'particles' independently drawn from the configuration with probability proportional to the particle mark, i.e., $C k$. Then, the (unconditional) probability of drawing some particle, which bears mark $k$, is $C k p_{k}$ with $C=1 / a$ since the total probability should sum up to one. As a result, each root neighbor has a degree $k$ with probability $\hat{p}_{k}$. In the same way one picks the next-neighbors of $o$. This procedure is continued ad infinitum until the whole tree is constructed. Notably, except for the root degree, the distribution of $k(x)$ is given by $\hat{p}$, whereas $p$ itself is the distribution of marks, i.e., auxiliary objects used in the graph construction.

\subsection{The proposed model}

Our model is a random tree, also consistent with the assumptions of [2, 11, 12]. As in the case of the Galton-Watson tree, we begin with the description of the corresponding branching process. Let $p, \hat{p}$, and $a$ be as in (2.1) and (2.5). An additional parameter is $s \in \mathbb{N}$. The whole population falls into two types 'distinguished' and 'ordinary' individuals. At the beginning, there are no ordinary individuals and there is one distinguished individual, who independently produces $m$ offsprings with probability $\hat{p}_{m}$. If $m \leqslant s$, all of them are set to be distinguished. For $m>s, s$ the offsprings are distinguished, and the remaining $m-s$ ones are set to be ordinary. Each ordinary individual independently produces $k$ ordinary individuals with probability $p_{k+1}$. Each distinguished individual, other than the initial one, independently produces $m$ offsprings with probability $\hat{p}_{m+1}$. As above, if $m \leqslant s$, then all of the offsprings are distinguished. For $m>s, s$ offsprings are distinguished and the remaining $m-s$ ones are ordinary. This process is repeated ad infinitum. For $n \in \mathbb{N}_{0}$, by $\widehat{L}_{n}$ and $L_{n}$ we denote the number of distinguished and ordinary individuals in generation $n$, respectively. Let also $\widehat{X}$ and $X$ be independent and such that $\operatorname{Prob}(\widehat{X}=m)=\hat{p}_{m+1}$ and $\operatorname{Prob}(X=m)=p_{m+1}, m \in \mathbb{N}$. Then, we have, cf. (2.3),

$$
\widehat{L}_{n}=\sum_{j=1}^{\widehat{L}_{n-1}}\left[\widehat{X}_{n, j}-\left(\widehat{X}_{n, j}-s\right)_{+}\right], \quad L_{n}=Y_{n}+\sum_{j=1}^{L_{n-1}} X_{n, j},
$$

where

$$
Y_{n}=\sum_{j=1}^{\widehat{L}_{n-1}}\left(\widehat{X}_{n, j}-s\right)_{+},
$$

and for an integer $\varkappa$, we write $\varkappa_{+}=\max \{0 ; \varkappa\}$, whereas $\widehat{X}_{n, j}$ and $X_{n, j}$ are independent copies of $\widehat{X}$ and $X$, respectively. From the point of view of ordinary particles, those represented by the first term in the expression for $L_{n}$ are immigrants. Then, the total number of individuals in the generation $n$ can be written in the form

$$
\widetilde{L}_{n}:=\widehat{L}_{n}+L_{n}=\sum_{j=1}^{\widehat{L}_{n-1}} \widehat{X}_{n, j}+\sum_{j=1}^{L_{n-1}} X_{n, j} .
$$

Since each distinguished individual can have at most $s$ distinguished offsprings, we have that

$$
\widehat{L}_{n} \leqslant s^{n} \text {. }
$$

\footnotetext{
${ }^{3}$ Also models which locally converge to $\mathrm{T}(P, \rho, \infty)$, cf. Definition 2.1 in [9].

${ }^{4}$ In [2], a mark is the number of stubs.
} 
The graph in question is the genealogic tree of the process just described. Its construction can be visualized as follows. The graph is a rooted tree with $\operatorname{Prob}(k(o)=k)=\hat{p}_{k+1}$. Let $k(o)=m \leqslant s$. Then, we draw $m$ neighbors of $o$ as in the configuration model. Denote them $x_{1}, \ldots, x_{m}$; by construction $\operatorname{Prob}\left(k\left(x_{l}\right)=\right.$ $k)=\hat{p}_{k}$ for all $l=1, \ldots m$. If $m>s$, we draw $s$ neighbors $x_{1}, \ldots, x_{s}$ of $o$ as in the configuration model. The remaining neighbors $y_{s+1}, \ldots, y_{m}$ are set to be the roots of independent Galton-Watson trees with probability distribution $p$, which in particular means that $\operatorname{Prob}\left(k\left(y_{l}\right)=k\right)=p_{k}, l=s+1, \ldots, m$. This procedure is continued ad infinitum. For $s>1$, the obtained tree contains a subtree, comprised by an infinite number of distinguished rays $\left\{o, x_{1}, x_{2}, \ldots, x_{n}, \ldots\right\}$ with the mentioned path property "the neighbor of $x_{n-1}$ has a degree $k$ with probability $\hat{p}_{k}$ ". The set of vertices of this subtree is contained in that of a Cayley tree with root $o$ and branching number $s$. For $s=1$, our graph is a size-biased Galton-Watson tree, see [6, pp. 407-412] or [15], in which there is only one distinguished ray.

In the remaining part of the article we use the following nomenclature. By $\mathbf{G W}(s, p), s \in \mathbb{N}_{0}$, we denote the model constructed in this subsection. Then, $\mathbf{G W}(1, p)$ and $\mathbf{G W}(0, p)$ are the size-biased and the ordinary Galton-Watson trees, respectively.

\section{The thermodynamic states of the Ising model}

\subsection{The thermodynamic states}

Let $\mathrm{G}=(\mathrm{V}, \mathrm{E})$ be a general tree with root $o$. Given $x \in \mathrm{V}$, the distance $\rho(o, x)$ is the length of the path $\vartheta(o, x)$. For $n \in \mathbb{N}_{0}$, by $\mathrm{S}_{n}$ we denote the set of vertices $x$ such that $\rho(o, x)=n$. Put

$$
\mathrm{V}_{n}=\bigcup_{m=0}^{n} \mathrm{~S}_{m}
$$

and let $\mathrm{E}_{n}$ be the set of all edges with both ends in $\mathrm{V}_{n}$. Let also $\mathrm{E}_{n}^{b}$ be the set of $\{x, y\}$ such that $x \in \mathrm{S}_{n}$ and $y \in \mathrm{S}_{n+1}$.

In the sequel, we assume that

$$
J:=\sup _{\{x, y\} \in \mathrm{E}}\left|J_{x y}\right|<\infty
$$

By $\sigma_{n}$ we denote the configuration of spins in $\mathrm{V}_{n}$, that is, $\sigma_{n}=\left\{\sigma_{x}: x \in \mathrm{V}_{n}\right\}$. The Gibbs probability distribution of configurations $\sigma_{n}$ at temperature $T$ and $h=0$ is the following probability measure

$$
\pi_{n}\left(\sigma_{n} \mid \xi\right)=\frac{1}{Z_{n}(\xi)} \exp \left[\sum_{\{x, y\} \in \mathrm{E}_{n}}\left(K_{x y} \sigma_{x} \sigma_{y}+K\right)+\sum_{\{x, y\} \in \mathrm{E}_{n}^{b}} K_{x y} \sigma_{x} \xi_{y}\right],
$$

where

$$
K_{x y}:=\beta J_{x y}, \quad K:=\beta J, \quad \beta:=1 / k_{\mathrm{B}} T .
$$

Note that $K_{x y}$ can have arbitrary signs, whereas $K$ is positive. In (3.2), the first (respectively the second) summand in $\exp (\cdots)$ corresponds to the interaction of the spins in $\mathrm{V}_{n}$ with each other (respectively with the spins $\xi_{y}$ fixed outside $\mathrm{V}_{n}$ ). One observes that the latter interaction involves only $\xi_{y}$ with $y \in \mathrm{S}_{n+1}$. For technical reasons, we add positive constants to the spin-spin interaction along each edge in $\mathrm{E}_{n}$, cf. (1.1). The partition function is then

$$
Z_{n}(\xi)=\sum_{\sigma_{n}} \exp \left[\sum_{\{x, y\} \in \mathrm{E}_{n}}\left(K_{x y} \sigma_{x} \sigma_{y}+K\right)+\sum_{\{x, y\} \in \mathrm{E}_{n}^{b}} K_{x y} \sigma_{x} \xi y\right],
$$

where the summation is taken over all $\sigma_{x}= \pm 1, x \in \mathrm{V}_{n}$.

Given $z \in \mathrm{V}$, let $n_{z} \in \mathbb{N}_{0}$ be such that $z \in \mathrm{S}_{n_{z}}$, i.e., $n_{z}=\rho(o, z)$. For these $z, n_{z}$, and for $n>n_{z}$,

$$
M_{n, z}(\xi)=\sum_{\sigma_{n}} \sigma_{z} \pi_{n}\left(\sigma_{n} \mid \xi\right)=\sum_{\sigma_{z}= \pm 1} \sigma_{z} \varrho_{n, z}\left(\sigma_{z} \mid \xi\right)=\varrho_{n, z}(1 \mid \xi)-\varrho_{n, z}(-1 \mid \xi)
$$


is the magnetization at $z \in \mathrm{V}_{n}$ in state $\pi_{n}(\cdot \mid \xi)$ 3.2. Here, for $\sigma_{z}=\alpha$,

$$
\varrho_{n, z}(\alpha \mid \xi)=\sum_{\sigma_{n}: \sigma_{z}=\alpha} \pi_{n}\left(\sigma_{n} \mid \xi\right), \quad \alpha= \pm 1
$$

where the summation is taken over all $\sigma_{n}$ with the fixed $\sigma_{z}=\alpha$. According to the theory of Gibbs states [1], the global thermodynamic state is unique if, for all $z$, the limits

$$
\varrho_{z}(\alpha):=\lim _{n \rightarrow+\infty} \varrho_{n, z}(\alpha \mid \xi)
$$

exist and are independent of $\xi$. In this case, by (3.5) the global magnetization $M_{z}$ exists and

$$
M_{z}=\lim _{n \rightarrow+\infty} M_{n, z}(\xi)=\varrho_{z}(1)-\varrho_{z}(-1)
$$

As $h=0$, we have $M_{n, z}(\xi)=-M_{n, z}(-\xi)$, and hence $M_{z}=-M_{z}$. Therefore, the uniqueness of thermodynamic states occurs if and only if, for all $z \in \mathrm{V}$ and $\xi$,

$$
M_{z}=\lim _{n \rightarrow+\infty} M_{n, z}(\xi)=0
$$

In this case, we say that the model is in a paramagnetic state.

If the underlying tree $\mathrm{G}$ is random, then the measure (3.2) is also random, and hence (3.8) is a random event obeying the zero-one law. If $\operatorname{Prob}\left(M_{z}=0\right)=1$, we say that the model (1.1) on $\mathrm{G}$ is in a paramagnetic state with probability one.

\subsection{The main statement}

Let $p$ and $\hat{p}$ be as in 2.1) and (2.5). For $\alpha \in(0,1)$, we set

$$
b_{\alpha}=\sum_{k \geqslant s+1}(k-s)^{\alpha} \hat{p}_{k}, \quad b=\sum_{k \geqslant 2} k p_{k} \ln k,
$$

and also

$$
K_{\mathrm{c}}=\frac{1}{4} \ln \frac{a}{a-1}, \quad \widehat{K}_{c}=\frac{1}{4} \ln \frac{s^{\gamma}+1}{s^{\gamma}}, \quad \gamma=1 / \alpha .
$$

In the statement below we describe the Ising model (1.1) on $\mathbf{G W}(s, p)$ graphs with $p$ obeying (2.1).

Theorem 3.1 For $s=0$, the Ising model is in a paramagnetic state whenever $K<K_{\mathrm{c}}$. For $s=1$, the same holds under the condition $b<\infty$. For $s \geqslant 2$, the Ising model is in a paramagnetic state whenever $b_{\alpha}<\infty$ for some $\alpha \in(0,1)$ and $K<\min \left\{K_{\mathrm{c}} ; \widehat{K}_{c}\right\}$.

In section 5. we discuss the above statement in detail. Its proof is based on two lemmas proven in the next section. In the first lemma, we describe the model (1.1) on a general tree, for which the corresponding quantities were introduced in subsection 3.1 .

Lemma 3.2 Let $K>0$ be such that

$$
q(K):=\exp (4 K)-1<1
$$

Then, for each $z \in \mathrm{V}$, any $n>n_{z}$, and arbitrary $\xi$ and $\eta$, we have that

$$
\left|M_{n, z}(\xi)-M_{n, z}(\eta)\right| \leqslant 2[q(K)]^{n-n_{z}}\left|\mathrm{~S}_{n}\right|
$$

In the next lemma, we describe the model $\mathbf{G W}(s, p)$ introduced in subsection 2.3

Lemma 3.3 Let $L_{n}$ be as in (2.7), $s \geqslant 2$, and $b_{\alpha}<\infty$ for some $\alpha \in(0,1)$. Then, for every $c$ obeying the conditions $c>s^{\gamma}$ and $c \geqslant a-1$, with probability one

$$
c^{-n} L_{n} \rightarrow W \in[0, \infty), \quad \text { as } n \rightarrow \infty
$$


Proof of theorem 3.1. For $\underline{s=0}$, by construction $\left|S_{n}\right|=L_{n}$ is the number of individuals in generation $n$, see (2.3), and $a-1$ is the mean number of offsprings in the corresponding Galton-Watson process. Then, the limit

$$
\lim _{n \rightarrow+\infty} \frac{L_{n}}{(a-1)^{n}}
$$

with probability one exists and is finite, see (2.2) and proposition 1.3 in [5, page 20]. If $K<K_{\mathrm{c}}$, then $q(K)<q\left(K_{\mathrm{c}}\right)=1 /(a-1)<1$, and hence we can apply (3.12), which yields that with probability one

$$
[q(K)(a-1)]^{n} \frac{L_{n}}{(a-1)^{n}} \rightarrow 0, \quad n \rightarrow+\infty,
$$

which in turn by (3.12) yields (3.8), and hence the proof for this case.

For $\underline{s=1}$, by 2.10 we have $\widehat{L}_{n}=1$, and hence $\left|S_{n}\right|=\widetilde{L}_{n}=1+L_{n}$. Since $b<\infty$, with probability one we have

$$
\sum_{n \geqslant 1}(a-1)^{-n} Y_{n}<\infty
$$

see proposition 6.2 in [5, page 50]. Then, by theorem 6.1 in [5, page 50], $\widetilde{L}_{n} /(a-1)^{n}$ with probability one tends to a finite limit. Then, the proof follows as in the case of $s=0$.

For $s \geqslant 2$, the random variables $Y_{n}$ with different $n$ are no more identically distributed, and hence $b<\infty$ is not sufficient to get (3.15). Instead, we use a more restrictive condition $b_{\alpha}<\infty$, under which we get, see lemma 3.3. that $c^{-n}\left|\mathrm{~S}_{n}\right|=c^{-n} \widetilde{L}_{n}=c^{-n} \widehat{L}_{n}+c^{-n} L_{n}$ with probability one tends to a finite limit since $c>s$, cf. (2.10). Note that

$$
K(c):=\frac{1}{4} \ln \frac{c+1}{c} \leqslant \min \left\{K_{c} ; \widehat{K}_{c}\right\} .
$$

Hence, for $K<K(c)$, we have $q(K)<1 / c$, and the proof follows as in (3.14).

\section{The proof of the lemmas}

\subsection{The proof of lemma 3.2}

Let $\mathrm{G}=(\mathrm{V}, \mathrm{E})$ be a general tree. For a path $\vartheta$, by $\mathrm{E}_{\vartheta}$ we denote the set of edges $\left\{x_{l-1}, x_{l}\right\}$ of $\vartheta$. Since $\mathrm{G}$ is a tree, there exists exactly one path $\vartheta(x, y)$ for any $x$ and $y$. Recall that $\mathrm{E}_{n}$ stands for the set of edges with both ends in $\mathrm{V}_{n}$. Thus, $\mathrm{G}_{n}=\left(\mathrm{V}_{n}, \mathrm{E}_{n}\right)$ is a finite graph; $\mathrm{G}^{\prime}=\left(\mathrm{V}^{\prime}, \mathrm{E}^{\prime}\right)$ is called a subgraph of $\mathrm{G}_{n}$ if $\mathrm{V}^{\prime} \subset \mathrm{V}_{n}$ and $\mathrm{E}^{\prime} \subset \mathrm{E}_{n}$. A path $\vartheta$ is said to be in $\mathrm{G}^{\prime}$ if $\mathrm{E}_{\vartheta} \subset \mathrm{E}^{\prime}$. Two vertices $z, x \in \mathrm{V}^{\prime}$ are said to be disconnected in $\mathrm{G}^{\prime}$ if there is no path $\vartheta(z, x)$ in $\mathrm{G}^{\prime}$.

By (3.5) and (3.2), employing replica spins $\tilde{\sigma}$ we obtain the following Meyer-like expansion

$$
\begin{aligned}
M_{n, z}(\xi)-M_{n, z}(\eta) & =\sum_{\sigma_{n}, \tilde{\sigma}_{n}}\left(\sigma_{z}-\tilde{\sigma}_{z}\right) \pi_{n}\left(\sigma_{n} \mid \xi\right) \pi_{n}\left(\tilde{\sigma}_{n} \mid \eta\right) \\
& =\frac{1}{Z_{n}(\xi) Z_{n}(\eta)} \sum_{\sigma_{n}, \tilde{\sigma}_{n}}\left(\sigma_{z}-\tilde{\sigma}_{z}\right) \prod_{\{x, y\} \in \mathrm{E}_{n}}\left(1+\Gamma_{x y}\right) \Psi_{n}(\xi, \eta) \\
& =\frac{1}{Z_{n}(\xi) Z_{n}(\eta)} \sum_{\mathrm{E}^{\prime} \subset \mathrm{E}_{n}} \sum_{\sigma_{n}, \tilde{\sigma}_{n}}\left(\sigma_{z}-\tilde{\sigma}_{z}\right) \Gamma\left(\mathrm{E}^{\prime}\right) \Psi_{n}(\xi, \eta),
\end{aligned}
$$

where

$$
\begin{aligned}
\Gamma\left(\mathrm{E}^{\prime}\right) & =\prod_{\{x, y\} \in \mathrm{E}^{\prime}} \Gamma_{x y}, \\
\Gamma_{x y} & =\exp \left[\left(K_{x y} \sigma_{x} \sigma_{y}+K\right)+\left(K_{x y} \tilde{\sigma}_{x} \tilde{\sigma}_{y}+K\right)\right]-1, \\
\Psi_{n}(\xi, \eta) & =\prod_{\{x, y\} \in \mathrm{E}_{n}^{b}} \exp \left[K_{x y}\left(\sigma_{x} \xi_{y}+\tilde{\sigma}_{x} \eta_{y}\right)\right] .
\end{aligned}
$$


For simplicity, we do not indicate the dependence of the latter functions on the spins. One observes that each $\Gamma_{x y} \geqslant 0$ due to our choice of the spin-spin interactions in (3.2), cf. (3.1), and that $\Psi_{n}(\xi, \eta)$ depends only on $\sigma_{x}$ and $\tilde{\sigma}_{x}$ with $x \in \mathrm{S}_{n}$. Fix some $\mathrm{E}^{\prime}$ in the last line in (4.1) and consider the subgraph $\mathrm{G}^{\prime}$ with the edge set $\mathrm{E}^{\prime}$ and the vertex set $\mathrm{V}_{n}$. If in $\mathrm{G}^{\prime}$, the vertex $z$ is disconnected from each $x \in \mathrm{S}_{n}$, then the sums over the spins $\sigma_{z}, \tilde{\sigma}_{z}$ and over $\sigma_{x}, \tilde{\sigma}_{x}$ with $x \in \mathrm{S}_{n}$ get independent and hence the left-hand side of (4.1) vanishes as the term $\left(\sigma_{z}-\tilde{\sigma}_{z}\right)$ is antisymmetric with respect to the interchange $\sigma \leftrightarrow \tilde{\sigma}$, whereas all $\Gamma_{x y}$ are symmetric and the only break of this symmetry is related to the fixed boundary spins $\xi(y)$ and $\eta(y)$, $y \in \mathrm{S}_{n+1}$. Therefore, each non-vanishing term in (4.1) corresponds to a path $\vartheta(z, x)$ connecting $z$ to some $x \in \mathrm{S}_{n}$. Let us take this into account and rewrite (4.1) as the sum over the subsets of $\mathrm{E}_{n}$ containing the edges of at least one such path. Let $\Theta_{n}(z)$ be the set of all paths connecting $z$ to $S_{n}$, and for $\vartheta \in \Theta_{n}(z)$, let $\mathscr{E}_{\vartheta}$ be the family of subsets of $\mathrm{E}_{n}$ each of which contains $\mathrm{E}_{\vartheta}$. That is, $\mathscr{E}_{\vartheta}=\left\{\mathrm{E}^{\prime} \subset \mathrm{E}_{n}: \mathrm{E}_{\vartheta} \subset \mathrm{E}^{\prime}\right\}$. Note that, for distinct $\vartheta, \vartheta^{\prime} \in \Theta_{n}(z)$, the corresponding families $\mathscr{E}_{\vartheta}$ and $\mathscr{E}_{\vartheta^{\prime}}$ are not disjoint - they include those $E^{\prime}$ which contain both $\mathrm{E}_{\vartheta}$ and $\mathrm{E}_{\vartheta^{\prime}}$. Finally, set

$$
\mathscr{E}=\bigcup_{\vartheta \in \Theta_{n}(z)} \mathscr{E}_{\vartheta}
$$

that is, $\mathscr{E}$ contains all sets of edges $\mathrm{E}^{\prime} \subset \mathrm{E}_{n}$ such that the corresponding graph $\mathrm{G}^{\prime}$ contains at least one path connecting $z$ to some $x \in \mathrm{S}_{n}$. Then, (4.1) takes the form

$$
M_{n, z}(\xi)-M_{n, z}(\eta)=\frac{1}{Z_{n}(\xi) Z_{n}(\eta)} \sum_{\mathrm{E}^{\prime} \in \mathscr{E}} \sum_{\sigma_{n}, \tilde{\sigma}_{n}}\left(\sigma_{z}-\tilde{\sigma}_{z}\right) \Gamma\left(\mathrm{E}^{\prime}\right) \Psi_{n}(\xi, \eta)
$$

In view of the positivity of all $\Gamma_{x y} \geqslant 0$, this yields

$$
\begin{aligned}
\left|M_{n, z}(\xi)-M_{n, z}(\eta)\right| & \leqslant \frac{2}{Z_{n}(\xi) Z_{n}(\eta)} \sum_{\mathrm{E}^{\prime} \in \mathscr{E}} \sum_{\sigma_{n}, \tilde{\sigma}_{n}} \Gamma\left(\mathrm{E}^{\prime}\right) \Psi_{n}(\xi, \eta) \\
& \leqslant \frac{2}{Z_{n}(\xi) Z_{n}(\eta)} \sum_{\sigma_{n}, \tilde{\sigma}_{n}} \sum_{\vartheta \in \Theta_{n}(z)} \Gamma\left(\mathrm{E}_{\vartheta}\right) \sum_{\mathrm{E}^{\prime} \in \mathscr{E}_{\vartheta}} \Gamma\left(\mathrm{E}^{\prime} \backslash \mathrm{E}_{\vartheta}\right) \Psi_{n}(\xi, \eta)
\end{aligned}
$$

Note that the sum $\sum_{\vartheta \in \Theta_{n}(z)} \sum_{\mathrm{E}^{\prime} \in \mathscr{E}_{\vartheta}}$ contains the same summands as $\sum_{\mathrm{E}^{\prime} \in \mathscr{E}}$, see (4.3), but a part of them are repeated, which yields the second $\leqslant$ in 4.5 . From (4.2) and (3.11) we see that $0 \leqslant \Gamma_{x y} \leqslant q(K)$, for any $x, y \in \mathrm{V}$. Hence,

$$
\Gamma\left(\mathrm{E}_{\vartheta}\right) \leqslant[q(K)]^{\|\vartheta\|}
$$

which yields in (4.5)

$$
\begin{aligned}
\left|M_{n, z}(\xi)-M_{n, z}(\eta)\right| \leqslant & \frac{2}{Z_{n}(\xi) Z_{n}(\eta)} \sum_{\vartheta \in \Theta_{n}(z)}[q(K)]^{\|\vartheta\|} \\
& \times \sum_{\sigma_{n}, \tilde{\sigma}_{n}} \prod_{\{x, y\} \in \mathrm{E}_{\vartheta}}\left(1+\Gamma_{x y}\right) \sum_{\mathrm{E}^{\prime \prime} \subset \mathrm{E}_{n} \backslash \mathrm{E}_{\vartheta}} \Gamma\left(\mathrm{E}^{\prime \prime}\right) \Psi_{n}(\xi, \eta) \\
= & 2 \sum_{\vartheta \in \Theta_{n}(z)}[q(K)]^{\|\vartheta\|} .
\end{aligned}
$$

Here, we have taken into account that, see [4.1,

$$
\sum_{\sigma_{n}, \tilde{\sigma}_{n}} \prod_{\{x, y\} \in \mathrm{E}_{\vartheta}}\left(1+\Gamma_{x y}\right) \sum_{\mathrm{E}^{\prime \prime} \subset \mathrm{E}_{n} \backslash \mathrm{E}_{\vartheta}} \Gamma\left(\mathrm{E}^{\prime \prime}\right) \Psi_{n}(\xi, \eta)=\sum_{\sigma_{n}, \tilde{\sigma}_{n}} \prod_{\{x, y\} \in \mathrm{E}_{n}}\left(1+\Gamma_{x y}\right) \Psi_{n}(\xi, \eta)=Z_{n}(\xi) Z_{n}(\eta) .
$$

Note that a similar construction was used in deriving (2.16) and (2.17) in [16], see also lemma 3.1 in [13]. Since $n_{z}$ is defined by the condition $z \in \mathrm{S}_{n_{z}}$, the shortest path in $\Theta_{n}(z)$ has length $n-n_{z}$. Furthermore, for every $x \in \mathrm{S}_{n}$, there exists exactly one path $\vartheta \in \Theta_{n}(z)$. Then, for $q(K) \leqslant 1$, the estimate (3.12) follows from 4.6. 


\subsection{The proof of lemma 3.3}

If we prove that, for $c>s^{\gamma}$, with probability one

$$
\sum_{n \geqslant 1} c^{-n} Y_{n}<\infty
$$

see (2.8), then the proof that $L_{n} / c^{n} \rightarrow W \in[0,+\infty)$, with probability one, follows as in the proof of theorem 6.1 in [5, page 50]. The first summand of $L_{n}$ in [2.7) is under control in view of the assumed property $c \geqslant a-1$.

As $\alpha \in(0,1)$, by the standard Minkowski inequality, cf. [17, theorem 8, page 319], as well as by 2.10 , and [3.9], we have

$$
\left\langle Y_{n}^{\alpha}\right\rangle \leqslant \sum_{j=1}^{s^{n-1}}\left\langle\left(\widehat{X}_{j}-s\right)_{+}^{\alpha}\right\rangle=s^{n-1} b_{\alpha} .
$$

Then, for any $q>c^{\gamma}$, by Markov's inequality, cf. [17, page 311],

$$
P_{n}:=\operatorname{Prob}\left(q^{-n} Y_{n}>1\right)=\operatorname{Prob}\left(Y_{n}^{\alpha}>q^{\alpha n}\right) \leqslant \frac{b_{\alpha}}{s}\left(\frac{s}{q^{\alpha}}\right)^{n} .
$$

On the other hand, for $c>s^{\gamma}$, we take $q \in\left(s^{\gamma}, c\right)$ and rewrite

$$
\sum_{n \geqslant 1} c^{-n} Y_{n}=\sum_{n \geqslant 1}\left(\frac{q}{c}\right)^{n}\left(q^{-n} Y_{n}\right) .
$$

For such $q$, by (4.8) we have that $\sum_{n \geqslant 1} P_{n}<\infty$. Thus, the Borel-Cantelli lemma, cf. [17, page 320], yields that with probability one, only finitely many of the events $q^{-n} Y_{n}>1$ may occur, which by (4.9) readily yields 4.7 .

\section{Concluding comments and remarks}

\subsection{The graph model}

Let us look at the structure of the generation $n$ in $\mathbf{G W}(s, p)$, cf. (2.7) - 2.10). For $s=1$, we deal with a size-biased Galton-Watson tree, cf. [6] and [15], and $b<\infty$ is known as the $X \log X$ condition of the KestenStigum theorem, see also [5, page 23]. If it holds, the effect of the distinguished ray on the structure of $S_{n}$ is asymptotically negligible, see section 3 in [15]. For $s \geqslant 2$, the number of distinguished vertices $\widehat{L}_{n}$ in the generation $n$ increases in such a way that the sequence $\left\{\sigma^{-n} \widehat{L}_{n}\right\}_{n \in \mathbb{N}}$ with probability one tends to a random variable $\widehat{W}$ such that $\operatorname{Prob}(\widehat{W}>0)=1$ and $\langle\widehat{W}\rangle=1$. Here,

$$
\sigma=s-(s-1) \hat{p}_{2}-(s-2) \hat{p}_{3}-\cdots-\hat{p}_{s} .
$$

Note that $\sigma \in(1, s]$, and $\sigma=s$ if and only if $p_{2}=\cdots=p_{s}=0$, and hence the least value of $X$ is $s$. In this case, $a-1>s$. If $a-1>s^{\gamma}, L_{n}$ asymptotically 'behaves' like in the ordinary Galton-Watson tree, which means that the production of offsprings with probability $p$ 'dominates' the immigration from the distinguished part of the population, which is always the case for $\mathbf{G W}(1, p)$, cf. (2.2). However, for $a-1 \leqslant s^{\gamma}$, the main contribution into $L_{n}$ comes from the immigration. If $a-1$ is close to one and $s^{\gamma} \gg 1$, even for big $n$ the structure of $\left(\mathrm{V}_{n}, \mathrm{E}_{n}\right)$ is quite close to that of the corresponding subgraph of $\mathbf{G W}(0, \hat{p})$, i.e., as in the case of the configuration model. Note that $s^{\gamma}$ is big whenever (a) $s$ is big, and hence the distinguished subgraph is 'big'; (b) $\alpha$ is small, and hence the offspring production in the distinguished subgraph is very intensive.

Noteworthy, the procedure of formation of edges in $\mathbf{G W}(s, p)$ resembles that of [7, 8]. The 'value' specified in [8] by a positive $\omega$, in our case appears as a mark $k$, distributed according to $p$ with $p_{k}$ 'decreasing at infinity', cf. "inverse mass-action principle” of [8]. Distinguished individuals have the right to choose neighbors. Each chooses according to its own mark at most $s$ distinguished neighbors, preferring those with big marks. The restriction of the number of distinguished neighbors to $s$ corresponds to the restriction of the number of outgoing edges in the Cameo principle [8]. 
One observes that $\mathbf{G W}(s, p)$ is a natural generalization of the size-biased Galton-Watson tree $\mathbf{G W}(1, p)$. In a separate work, we plan to study this model in more detail. Another generalization of $\mathbf{G W}(1, p)$ can be the model in which the distinguished part of $\widehat{X}$ is not $\widehat{X}-(\widehat{X}-s)_{+}$like in $\mathbf{G W}(s, p)$, cf. (2.7), but rather it is a more general increasing function of $\widehat{X}$, or it is random. Further generalizations of this kind can be obtained in the approach of [18].

\subsection{Thermodynamic states}

In 1989, R. Lyons [14] proved that, for any tree, the critical temperature of the model [1.1) with $h=0$ and $J_{x y}=J>0$ is

$$
T_{\mathrm{c}}=\frac{J}{k_{\mathrm{B}} \operatorname{coth}^{-1} \varrho},
$$

where $\varrho$ is the branching number of the underlying tree. For an infinite (almost surely non-extinct) GaltonWatson tree, with probability one $\varrho$ equals the mean number of offsprings, see proposition 6.4 in [19]. Hence, for $\mathbf{G W}(0, \hat{p})$, we have $\varrho=\left\langle k^{2}\right\rangle /\langle k\rangle-1$, which yields in 5.1)

$$
T_{\mathrm{c}}=2 J / k_{\mathrm{B}} \ln \frac{\left\langle k^{2}\right\rangle}{\left\langle k^{2}\right\rangle-2\langle k\rangle} .
$$

The very same expression for $T_{\mathrm{c}}$ was 'rediscovered' in 2002 in [11, 12]. It is significant that Lyons' paper [14] was quoted in [2] and even the same formula (5.1) was discussed, see the text between equations (89) and (90) on page 1304 in [2] or the text between equations (89) and (90) on page 33 in [20].

Now let us turn to the discussion of theorem 3.1 which describes the model (1.1) with arbitrary signs 6 of the intensities $J_{x y}$. The only condition is that they are uniformly bounded, cf. (3.1). As follows from the estimate (3.12), the model (1.1) on a general tree can be in a paramagnetic state if the number of vertices in the sphere $\mathrm{S}_{n}$ admits the control $\left|\mathrm{S}_{n}\right| \leqslant c^{n}$ for some $c>1$. Note that in the example of [13], $\left|\mathrm{S}_{n}\right| \sim n !$. For graphs other than tree 7 , one has to $\operatorname{control} \Theta_{n}(z)$, cf. (4.6). The results of theorem 3.1 can naturally be extended to models with continuous spins and bounded interaction, as, e.g., in [13]. In a separate work, we plan to study the case of unbounded interaction intensities, which also includes random $J_{x y}$, as, e.g., in the Edwards-Anderson model. For a ferromagnetic Ising model on a $\mathbf{G W}(0, p)$, the exact value of the critical temperature is given in (5.1) with $\varrho=a-1$. The corresponding result of theorem 3.1 is that the model is in a paramagnetic state for

$$
T>4 J / k_{\mathrm{B}} \ln \frac{a}{a-1}>T_{\mathrm{c}} .
$$

It is naturally less precise as we cover the case of arbitrary signs of $J_{x y}$.

Regarding the model $\mathbf{G W}(0, \hat{p})$ studied in [11, 12], by theorem 1.1 we get that the Ising model with arbitrary signs of $J_{x y}$ obeying $(3.1)$ is in a paramagnetic state if

$$
T>4 J / k_{\mathrm{B}} \ln \frac{\left\langle k^{2}\right\rangle}{\left\langle k^{2}\right\rangle-1} .
$$

It is believed, cf. [22], that the Internet can well be modeled as a scale-free graph with $\lambda=5 / 2$, which corresponds to the choice of $\alpha<1 / 2$, see (3.9). As a byproduct of the result of theorem 3.1 we obtain the following, cf. [22, 23],

Proposition 5.1 Let $\theta \in(0,1)$ be the Bernoulli bond percolation probability on $\mathbf{G W}(s, p)$ graphs with $s \geqslant 2$ and $p$ obeying (2.1) and $b_{\alpha}<\infty$. Then, with probability one there is no giant component if

$$
\theta<\min \left\{q\left(K_{\mathrm{c}}\right) ; q\left(\widehat{K}_{\mathrm{c}}\right)\right\},
$$

where $q(K)$ is given by 3.11, and $K_{\mathrm{c}}$ and $\widehat{K}_{\mathrm{c}}$ are as in theorem 1.1

\footnotetext{
${ }^{5}$ In the preprint version [20] of [2], both papers [14, 19] were quoted.

${ }^{6}$ The antiferromagnetic case $J_{x y}=-J, J>0$, can be reduced to the ferromagnetic one by changing signs of $\sigma_{x}, x \in \mathrm{S}_{2 n}$.

${ }^{7}$ For more on phase transitions on graphs see [21].
} 


\section{Acknowledgements}

The author benefited from the discussions on the matter of this work with Philippe Blanchard, Yurij Holovatch, and Yuri Kondratiev, for which he is cordially indebted. The work was supported in part by the DFG through SFB 701: "Spektrale Strukturen und Topologische Methoden in der Mathematik" and through the research project 436 POL 125/113/0-1.

\section{References}

1. Georgii H.-O., Gibbs Measures and Phase Transitions, de Gruyter Studies in Mathematics, Vol. 9, Walter de Gruyter, Berlin, New York, 1988.

2. Dorogovtsev S.N., Goltsev A.V., Mendes J.F.F., Rev. Mod. Phys., 2008, 80, 1275; doi 10.1103/RevModPhys.80.1275

3. Newman M.E.J., SIAM Rev., 2003, 45, 167; doi 10.1137/S003614450342480

4. Barabási A.-L., Science, 2009, 325, 412l; doi 10.1126/science.1173299.

5. Asmussen S., Hering H., Branching Processes, Progress in Probability and Statistics, Vol. 3, Birkhäuser Boston, Boston, 1983.

6. Lyons R., Peres Y., Probability on Trees and Networks, Cambridge University Press (in press, http://mypage.iu.edu/ rdlyons/prbtree/prbtree.html).

7. Blanchard Ph., Chang C.-H., Krüger T., Ann. Henri Poincaré, 2003, 4, No. 2 Suppl., S954; doi 10.1007/s00023-003-0975-1.

8. Blanchard Ph., Krüger T., J. Stat. Phys., 2004, 114, 1399; doi 10.1023/B:JOSS.0000013969.87579.39

9. Dembo A., Montanari A., Ann. Appl. Probab., 2010, 20, 565; doi 10.1214/09-AAP627

10. Dommers S., Giardinà C., van der Hofstad R., J. Stat. Phys., 2010, 141, 638; doi 10.1007/s10955-010-0067-9

11. Dorogovtsev S.N., Goltsev A.V., Mendes J.F.F., Phys. Rev. E, 2002, 66, 016104; doi 10.1103/PhysRevE.66.016104

12. Leone M., Vázquez A., Vespignani A., Zecchina R., Eur. Phys. J. B, 2002, 28, 191; doi 10.1140/epjb/e2002-00220-0.

13. Kepa D., Kozitsky Y., Condens. Matter Phys., 2008, 11, 313; doi 10.5488/CMP.11.2.313

14. Lyons R., Commun. Math. Phys., 1989, 125, 337; doi 10.1007/BF01217911.

15. Lyons R., Pemantle R., Peres Y., Ann. Probab., 1995, 23, 1125; doi 10.1214/aop/1176988176

16. Von Dreifus H., Klein A., Perez F.J., Commun. Math. Phys., 1995, 170, 21; doi 10.1007/BF02099437

17. Grimmett G., Stirzaker D., Probability and Random Processes, 3rd ed., Oxford University Press, New York, 2001.

18. Jagers P., Sagitov S., Bernoulli, 2008, 14, No. 4, 949; doi 10.3150/08-BEJ138

19. Lyons R., Ann. Probab., 1990, 18, 931; doi 10.1214/aop/1176990730

20. Dorogovtsev S.N., Goltsev A.V., Mendes J.F.F., Preprint arXiv:0705.0010v2 2007.

21. Lyons R., J. Math. Phys., 2000, 41, 1099; doi 10.1063/1.533179

22. Cohen R., Erez K., ben-Avraham D., Havlin S., Phys. Rev. Lett., 2000, 85, 4626; doi 10.1103/PhysRevLett.85.4626

23. Cohen R., ben-Avraham D., Havlin S., Phys. Rev. E, 2002, 66, 036113; doi 10.1103/PhysRevE.66.036113 


\title{
До термодинамічних станів моделі Ізинґа на безмасштабних ґрафах
}

\section{Ю. Козицький}

Інститут математики, Університет Марії Кюрі-Складовської, 20-031 Люблін, Польща

\begin{abstract}
Запропоновано модель безмасштабних випадкових ґрафів, яка є локально близькою до нескорельованих випадкових мереж з розбіжними $\left\langle k^{2}\right\rangle$, що вивчалася, наприклад, в статті Dorogovtsev S.N. et al., Rev. Mod. Phys., 2008, 80, 1275. Показано, що модель Ізинґа на запропонованих ґрафах з інтенсивностями взаємодії довільного знаку із ймовірністю один $\epsilon$ в парамагнітному стані для достатньо високих скінчених значень температури. Для таких ґрафів модель перколяції по ребрах з ймовірністю один $\epsilon$ в неперколятивному стані для позитивних значень перколяційної ймовірності. Обговорюються ці результати і можливості їх розширення.
\end{abstract}

Ключові слова: випадкові ґрафи, парамагнітна фаза, перколяція, процес розгалуження 